\title{
MJN THE CONTEXT OF POSTNATAL DEPRESSION IN MALAYSIA: AN OVERVIEW
}

\author{
Siti Roshaidai Mohd Arifin ${ }^{1^{*}}$, Helen Cheyne ${ }^{2}$, Margaret Maxwell ${ }^{2}$ \\ ${ }^{I}$ Department of Special Care Nursing, International Islamic University Malaysia, Kuantan, Pahang, \\ Malaysia \\ ${ }^{2}$ Nursing, Midwifery and Allied Health Professional (NMAHP) Research Unit, University of Stirling \\ Scotland, United Kingdom
}

*Corresponding Author's Email: roshaidai@iium.edu.my

\begin{abstract}
Sociocultural contexts of motherhood have been found to have a link with postnatal depression. Malaysia has a wide-range of cultural and ethnic backgrounds that offers a wonderful chance to understand the different role of cultures and postnatal practices in relation to postnatal depression. The purpose of this paper is to discuss the link between the Malaysian healthcare system, social and cultural context, the existing health policy and postnatal depression. This paper highlights that while there are different levels of nurses who work collaboratively to manage pregnant and postnatal mothers in the maternal and child health clinic, the focus of their care is more on physical health. The international guidelines for diagnosing PND were not commonly used within the clinics, although the Diagnostic and Statistical Manual of Mental Disorders (DSM-5) has been widely used within psychiatric setting in Malaysia. There is no formal mechanism or assessment to detect PND in the clinics. There were no guidelines found that specifically focused on the management of PND. It is questionable whether the current approaches to promote and manage maternal mental health in Malaysia have a sense of cultural-based strategies. These significant gaps call for empirical evidence that explicitly focussed on the experiences and perceptions of PND in Malaysia.
\end{abstract}

Keywords: Postnatal Depression, Malaysian Healthcare System, Pregnant Mothers

\section{INTRODUCTION}

The experience of postnatal depression (PND) is not fully shared by women across different cultures. Such experience can be influenced by societal and cultural interpretation and labelling of symptoms. Previous quantitative and qualitative studies have shown that there was a link between sociocultural factors and PND (Abdollahi, Etemadinezhad \& Lye, 2014; Gao et al., 2010). Within Asian culture, the traditional postnatal practice of staying inside the house for a month was described by the women as contributing to PND (Gao et al., 2010). The issue of gender preference of the baby and the existence of an 'external power' are two common factors linked to PND as reported by Asian women. Chinese and Indian women reported that they experienced conflict with their husbands and in-laws as they were blamed for not being able to give birth to a male child (Morrow et al., 2008; Gao et al., 2010). The existence of an 'external power' was particularly reported by South Asian and Chinese women (Edhborg Nasreen \& Kabir, 2015; Wittkowski et al., 2012).

The Malaysian population has a wide-range of cultural and ethnic backgrounds that offers a great opportunity to understand the different role of cultures and postnatal practices in relation to PND. Malaysia is a multi-ethnic country located in Southeast Asia. It covers an area of 329,847 square kilometres and has a total population of over 28.3 million (Department of Statistics Malaysia, 2010). Malaysian citizens comprise of three main races, including Malay (53.3\%), Chinese (26.0\%) and Indian (7.7\%) (Khader, 2012). Another 13\% of the Malaysian population is made up by other ethnic populations in Sabah and Sarawak (Ishak, 2009). While the Malays are regarded as the local or native population, the Chinese and Indian population in Malaysia today are descendants of those who originally migrated before the 
independence of Malaysia from Southern China and Southern India, respectively (Chee et al., 2005). The presence of the differences and similarities within Malaysian cultural backgrounds and associated traditions may affect the women's experience of PND (Fisher et al., 2004).

The purpose of this paper is to provide an overview of the Malaysian healthcare system and the sociocultural context and to understand how these elements were linked with the management of PND. Towards the end of this paper, a review of policy regarding management of PND in the Malaysian healthcare system is also presented.

\section{Healthcare System in Malaysia}

The healthcare system in Malaysia is comprised of a combination of both the private and public sector. Private clinics and hospitals are widely accessible in the urban areas and managed by non-government companies. The public sector is operated by the Ministry of Health $(\mathrm{MOH})$ and is the main healthcare service, encompassing a system of hospitals and primary care clinics. Hospital services provided by the $\mathrm{MOH}$ consist of small district hospitals in towns, to large hospitals in larger urban areas (Country Report, 2006). In the primary care settings, the $\mathrm{MOH}$ provides healthcare services under a two tier system, including small clinics (also known as community clinics) and larger community polyclinics (also known as health clinics).

Whilst community clinics provide healthcare services for approximately 2,000 to 4,000 mothers and children in rural areas (Jaafar et al., 2007), the health clinics offer a comprehensive range of services in larger towns and urban regions of approximately 15,000 to 20,000 persons (Ministry of Health Malaysia, 2012). Currently, health clinics often include a mix of the following health professionals: family medicine specialists; medical officers; physiotherapists; occupational therapists; nurses; assistant medical officers; nutritionists and dieticians (Mustapha et al., 2014). Maternal and child health (MCH) clinics are attached to the health clinics. Generally, these are managed by the nurses and a medical officer.

\section{Nursing and Midwifery in Malaysia}

There are several tiers of the nurses in Malaysia: community nurses, registered nurses, nurse-midwives, and head nurses. A community nurse is a person who has undergone a formal course of community nurse education (two years training in the community nursing college). Aregistered nurse is a person who has undergone a formal course of nursing education (either three years diploma or four years degree programme) and registered with the Malaysian Nursing Board (Malaysia Nursing Board, 2015). A nurse-midwife (or known as midwife in the UK) is a registered nurse who has successfully completed a one-year course of studies in midwifery and has acquired the requisite qualifications to be registered and legally licensed to practice midwifery (Ministry of Health Malaysia, 2013). This is contrast to the United Kingdom (UK) system, where midwives have their own separate training, and do not have to study nursing first. A head nurse is a registered nurse or a nurse-midwife who generally will have had 15 years working in clinical practices.

The different levels of nurses (community nurses, registered nurses, nurse-midwives and head nurses) work collaboratively to manage pregnant and postnatal mothers in the MCH clinic. They are trained and educated to provide healthcare services offered within the clinics. Such services are largely provided by the community nurses and nurse-midwives. In general, community nurses provide care for low risk mothers, while nurse-midwives provide care for high risk mothers. When it is necessary, they may refer an abnormal case to the head nurse and /or the medical doctor for further care and treatment.

Healthcare services offered within $\mathrm{MCH}$ clinics include antenatal, postnatal and child care (Country Report, 2006). In this first visit, community nurses or nurse-midwives perform antenatal assessment, family history of diseases. In subsequent visits, the community nurse or nurse-midwives conduct routine assessments. They also conduct the Oral Glucose Tolerance Test (OGTT) and assist in ultrasound scans when it is required (Baby Center, 2015).

Most women give birth in a maternity hospital, where they will be looked after during labour by nursemidwives, medical doctor and/ or obstetrician. Postnatal care includes home visits, breastfeeding guidance, and family planning, which is also delivered by the community nurses and/ or nurse midwives. Malaysian Ministry of Health guidelines for home visits are a total of eight visits, $1^{\text {st }}, 2^{\text {nd }}, 3^{\text {rd }}, 4^{\text {th }}, 6^{\text {th }}, 8^{\text {th }}, 10^{\text {th }}$ and $20^{\text {th }}$ postnatal days by the community nurses or nurse-midwives (Kaur 
\& Sigh, 2011). Child healthcare services covered by the community nurses or nurse-midwives include child immunization, child development monitoring up to four years of age, nutrition assessment and food supplement for those under-weight and home visits for high risk babies and malnourished children (Country Report, 2006).

In addition to the aforementioned roles, the nurses who work in the MCH clinics serve as link between the mothers and health services, educate and advise mothers within healthcare services and play an important role in the prevention of maternal mortality and morbidity and of premature, stillbirth and neonatal deaths (Manap, 1975).

The services offered by the $\mathrm{MCH}$ clinics play a major role in the promotive and preventive care for the urban and rural population. The referral system connects primary health care facilities with the hospitals (Ministry of Health Malaysia, 2008). Both health clinics and hospitals have common policies and operating procedures to facilitate management of referred cases. Community clinics are able to refer to health clinics or directly to the hospitals, according to the established patient-management protocols (Mustapha et al., 2014).

\section{Management of Perinatal Mental Health in Malaysia}

The focus of antenatal and postnatal care within the $\mathrm{MCH}$ clinics in Malaysia is more on physical health. $\mathrm{Ng}$ (2014) suggested that one of the reasons for the undiagnosed depressed cases in Malaysia is related to the Healthcare professionals (HCPs') over focus on physical issues. Such a focus results in less attention on the roles of managing mental health issues within their clinical practice. The international guidelines for diagnosing PND (e.g., DSM-5 and ICD-10) are not commonly used within the $\mathrm{MCH}$ clinics, although the DSM-5 has been widely used within psychiatric setting in Malaysia.

Yusuff et al., (2015) recommended that management and referral of depressed women should be included in Malaysian maternal health policy in order to prevent PND. Yet, there is no formal mechanism or assessment to detect PND in the $\mathrm{MCH}$ clinics. Although several screening tools have been translated into the Malay language with established psychometric properties (e.g., Beck Depression Inventory (BDI), Depression, Anxiety and Stress Scale (DASS), Edinburgh Postnatal
Depression Scale (EPDS), Hospital Anxiety and Depression Scale (HADS) and Patient Health Questionnaire 9 (PHQ-9), these are not routinely applied within the clinics (Mukhtar \& Oei, 2011). Rather, they have only generally been used in research studies on depression or PND.

Without a specific policy on screening and care for maternal mental health problems, antenatal or postnatal women with any mental health issues (including PND) are commonly referred from $\mathrm{MCH}$ clinic to the psychiatric unit in the hospital. When it is required, they are then treated within the same psychiatric setting as patients with general depression and other psychiatric problems.

The local guideline for managing general depression is known as Clinical Practice Guideline (CPG) for the Management of Depression (Ministry of Health Malaysia, 2007). While this local treatment guideline recommends both psychotherapy and pharmacotherapy ( $\mathrm{Ng}, 2014)$, the main form of treatment for depression in community settings and hospitals in Malaysia is pharmacological treatment (Razali \& Hasanah, 1999). In severe cases of puerperal psychosis, pharmacotherapy or electro-convulsive therapy is the main choice of treatment. Monotherapy with selective serotonin reuptake inhibitor is the first line of pharmacotherapy treatment recommended in the guideline $(\mathrm{Ng}, 2014)$.

Although psychotherapy for the treatment of depression is applied clinically in Malaysian clinical settings, there is less emphasis on the psychological aspects in the process of disease recognition and understanding, particularly for depression (Deva, 2006). There is also no empirical evidence to suggest that such use has been established and therefore it is still unknown whether psychological instruments for the assessment of depression and the theories for depression are valid and reliable for use in Malaysia (Mukhtar \& Oei, 2011). There is also less attention to primary prevention such as public education, awareness programmes and screening activities, although they have been recommended for all levels of the population (Yusuff et al., 2015; Ng, 2014).

\section{RESEARCH METHODOLOGY}

\section{Screening tools for postnatal depression}

As discussed in the previous section, the common assessment tools used to screen for depression in 
Malaysia are: BDI, DASS, EPDS and PHQ-9 (Mukhtar $\&$ Oei, 2011). These tools have been translated into the Malay language and their psychometric properties have been established (Mukhtar \& Oei, 2011).

The BDI is one of the most commonly used screening tools either in a clinical setting or in research on depression worldwide (Stahl, 2000). The use of BDI as an instrument to measure levels of depressive symptoms in Malaysia has also been established with the Cronbach's alpha between 0.56 to 0.90 and test-retest reliability between 0.56 to 0.87 , indicating acceptable reliability (Mukhtar \& Oei, 2011; Oei \& Mukhtar, 2008). The Depression Anxiety Stress Scale (DASS) is commonly used to screen for depression among general populations within outpatient clinics in Malaysia. The Cronbach's alpha and test-retest reliability for DASS-21 are between $0.74-0.83$ and $0.82-0.84$, respectively, indicating good reliability. The Cronbach's alpha of the PHQ-9 and HADS is 0.67 and test-retest reliability is 0.73, indicating good reliability (Mukhtar \& Oei, 2011).

While the above mentioned instruments have been validated within Malaysian population, they are commonly used to screen for general depressive symptoms. They were not specifically designed for screening for postnatal depressive symptoms.

EPDS was developed by Cox et al., (1987). It is a self-administered questionnaire designed specifically to measure the risk of PND (Cox et al., 1987). It consists of 10 items with four response scores of $0,1,2$, and 3 according to increased severity of the symptoms (Siti et al., 2014). The total score of EPDS ranges from 0 to 30 . The reliability and validity of the Malay version of the EPDS has been verified (Kadir et al., 2004; Wan Mohd Rushidi et al., 2003). It was found to have good internal consistency with Cronbach's alpha 0.86 and split half reliability with Spearman split half coefficient 0.83 (Wan Mohd Rushidi et al., 2003). The score of 11.5 represents the optimum cut-off point for $72.7 \%$ sensitivity, $95 \%$ specificity and positive predictive value of $80 \%$ (Kadir et al., 2004). As the cut off of $11 / 12$ was recommended to determine a woman at risk of developing PND (Yusuf et al., 2015; Wan Mohd Rushidi et al., 2003), women with an EPDS score $\geq 12$ were categorised as having depressive symptoms in this study.

The PHQ was developed by Spitzer et al., (1999). It is the most commonly used screening tool for the initial screening for major depression in primary care (worldwide) (Mitchell et al., 2016). The PHQ-9 consist of 9 questions regarding depression: 8 questions assess symptoms and 1 question assesses functional impairment. The questions are scored on a scale of 0 to 3 (Kroenke Spitzer \& Williams, 2001).The validity and reliability of the Malay version of the PHQ was conducted by Sherina Arroll \& Goodyear-Smith, (2012). The PHQ was found to be a valid and reliable instrument for detecting depression in primary care. Sherina Arroll \& Goodyear-Smith, (2012) suggested that the PHQ-9 had a sensitivity of $87 \%$ (95\% confidence interval $71 \%$ to $95 \%$ ), a specificity of $82 \%$ ( $74 \%$ to $88 \%$ ), positive Likelihood Ratio (LR) of 4.8 (3.2 to 7.2) and negative LR of 0.16 (0.06 to 0.40 ). The sensitivity of the PHQ-9 at the cut-off value of 10 and above was $87 \%$ and the specificity was $82 \%$.

\section{RESULTS AND DISCUSSION}

\section{The sociocultural context of postnatal depression in Malaysia}

\section{Postnatal practices in Malaysia}

There are slight differences in the postnatal practices among the three main cultures in Malaysia. However, they share several similarities in terms of the key elements used in the postnatal practice, dietary restrictions, and companions for the postnatal period.

Within the Malaysian communities, the postnatal period is commonly referred to as postnatal confinement. In Malay society, the postnatal period is called masa dalam pantang (Ministry of Health Malaysia, 2014) and both mother and baby are expected to remain housebound for around 44 days. In Chinese communities, the postnatal period is the point from the baby's birth up to one month later, whereas the postpartum period in the Indian community refers to the period after the childbirth until between 30 and 40 days later (Baby Center, 2014b).

The elements of herbs, heat and massage are traditionally practiced among newly delivered women in Malaysia, as a means of avoiding any health problems (Baby Center, 2014a). Among Malaysian communities, there is a practice of avoiding 'cooling food' such as cucumber and cabbage during postnatal confinement as these practices are said to enhance the blood circulation and toughen the joints of the mother (Baby Center, 2014a). 
In the postnatal period, most of the women will be taken care of by their own mother or mother-in-law. In this situation, some new mothers may have different views and beliefs from their caregivers, such as whether or not to follow restrictions in confinement diet and traditional practices of bathing and massage. Given that the sociocultural contexts of motherhood have a link with PND, an understanding of this concept in the Malaysian context may be of value (Buultjens \& Liamputtong, 2007).

\section{Studies on the sociocultural practices and postnatal depression in Malaysia}

In the past 30 years, Stern \& Kruckman (1983) have suggested that the incidence of PND was lower in Asian compared to western countries. Culturally based postnatal behavioural constraints were speculated as protecting Asian women from PND. However, with the changes from traditional to modern lifestyles in recent decades, this may no longer be the case in Malaysian society. In fact, some of the sociocultural elements have been highlighted in Malaysian as being contributing factors towards development of PND (Azidah et al., 2006; Grace et al., 2001).

Azidah et al., (2006) explored the association between sociocultural practices during the postnatal period and PND in Malaysia. They found that women undertaking traditional practices such as consuming traditional medication and practicing traditional massage were found to have 10-fold risk of getting PND. Azidah's findings corresponded to the earlier result by Grace et al., (2001) who studied postnatal women in Seremban General Hospital, Malaysia. Their findings indicated that a stronger adherence to traditional postnatal practices was linked with a higher depression score although this was not statistically significant.

Both researchers hypothesized that women with higher levels of PND were more likely to participate in traditional postnatal practices and/or that some element or substances in traditional practices may play a role in the development of PND. Furthermore, the Malaysian women were not allowed to leave the 'confinement house' during this period and they were expected to adhere to certain traditional practices to avoid unwelcomed consequences (Azidah et al., 2006). In this case, postnatal women probably suffered from depressed feelings without any assistance or referral to an appropriate channel. However, both studies used a quantitative design which may have limited the information on the nature and experience of PND in Malaysia.

\section{Policy related to postnatal depression in Malaysia}

The websites of the Ministry of Health $(\mathrm{MOH})$ Malaysia, the Malaysian Psychiatric Association, the Academy of Medicine Malaysia, and the Malaysian Mental Health Association were potential websites associated with healthcare in Malaysia. But no relevant documents were found on healthcare policy in the websites except from the website of the $\mathrm{MOH}$. A range of different documents were identified from this website, from clinical practice guidelines, health services policy and health bulletins. The search was restricted to 2000-2016 as it was felt this was related to the implementation of community mental health programme in Malaysia (which begins in 2000). Eleven documents were identified which were then read and summarised (Table 1). However, none of these were directly related to the management of PND.

\section{Table 1: Included policydocuments}

\begin{tabular}{|c|c|}
\hline Title & Year \\
\hline $\begin{array}{l}\text { Community Mental Health in Malaysia: Marriage of Psychiatry } \\
\text { and Public Health }\end{array}$ & 2000 \\
\hline Laws of Malaysia: Act 615- Mental Health Act 2001 & 2001 \\
\hline $\begin{array}{l}\text { Clinical Practice Guidelines: Management of Major Depressive } \\
\text { Disorders }\end{array}$ & 2007 \\
\hline Ministry of Health Malaysia Strategic Plan $2006-2010$ & 2008 \\
\hline $\begin{array}{l}\text { National Strategic Plan for Non-Communicable Disease (2010- } \\
\text { 2014) }\end{array}$ & 2010 \\
\hline General Hospital without Consultation-Liaison Psychiatrist & 2010 \\
\hline Psychiatric and Mental Health Services Operational Policy & 2011 \\
\hline Country Health Plan: $10^{\text {th }}$ Malaysia Plan 2011-2015 & 2011 \\
\hline $\begin{array}{l}\text { The Mental Health Development in Malaysia: History, Current } \\
\text { Issue and Future Development }\end{array}$ & 2013 \\
\hline Home Visiting & $\begin{array}{l}\text { No } \\
\text { Date }\end{array}$ \\
\hline $\begin{array}{l}\text { Traditional Postnatal Care in Restoring Women's Physical and } \\
\text { Mental Health Executive Summary }\end{array}$ & 2015 \\
\hline
\end{tabular}

The provision of mental health services in Malaysia is relatively new. In the early 1900s the mental health policy was based on British and Indian 
law (Chong Mohamad \& Er, 2013). In 2001, the National Mental Health Act detailed policy guidelines for the delivery of mental health services. This brought huge changes to the mental health system in Malaysia. Despite this achievement, it was argued that the act treats mental disorders as a medical problem, rather than a phenomenon that is triggered by a whole range of psychosocial factors within the larger society (Crabtree \& Chong, 2001).

A year before the act was enforced, community mental health was launched. This resulted in mental health care in Malaysia moving from hospital-based settings towards community-based care. There are two forms of community mental health services: formal and informal. Whilst formal services are linked with primary care services provided in a community, informal services are provided by local community members other than HCPs, including traditional healers, community support groups, and non-government organisations (Jamaiyah, 2000). Despite having a well-planned and comprehensive programme, the implementation of the community mental health service had faced several challenges. This included resistance to change from both psychiatrists and public health personnel. As some psychiatrists were hesitant to change from illness-oriented to wellnessoriented approaches, and some HCPs in the community were not involved with people who had mental disorders. There was also a lack of mental health practitioners (e.g., clinical psychologists, community mental health nurses), a lack of continuity care, problems in referral (especially for district hospitals without psychiatrists) and a lack of proper patient information flow (Jamaiyah, 2000).

Although all of the above challenges may not have been totally resolved, mental health care is currently integrated into all the health clinics, administratively under the public health division of the MOH. In 2010, in addition to psychiatric services in hospitals, 671 health clinics $(82.9 \%)$ provide mental health services in the community (Ministry of Health Malaysia, 2011).

Out of 11 the documents identified, only one document directly related to PND. This was a report of a review conducted to examine the effectiveness of traditional postnatal care. The review concluded that: (i) there were limited retrievable evidences to suggest the effectiveness of traditional postnatal care in restoring women's physical and mental health and (ii) there was no retrievable evidence on safety and costeffectiveness of traditional postnatal care (Akmal, 2015). Despite this conclusion, the Malay postnatal care package consisting of massage, hot compression (bertungku) and abdominal wrapping (barut), as well as postnatal breast massage, are still provided in the Traditional and Complementary Unit in several dedicated governmental hospitals in Malaysia.

In the absence of clinical practice guidelines for PND (or any form of maternal distress), the only source available is Clinical Practice Guidelines for Management of Major Depressive Disorders, which was introduced in 2007 (Ministry of Health Malaysia, 2007). However, it does not cover the management of depression in antenatal and postnatal women as stated in Figurel below:

TARGET POPULATION

This guideline is developed for the management of major depressive disorder in adults and the elderly. It does not cover the management of depression in children or pre- and post-natal women, or the management of dysthymic disorder, depressive episodes in patients with bipolar disorder or adjustment disorder with depressed mood

(Source: Ministry of Health Malaysia 2007)

Figure 1: Target population of clinical practice guidelines for management of major depressive disorders

\section{CONCLUSION}

This paper highlights that the focus of the current cares for antenatal and postnatal women within maternal and child health clinics are more on physical health rather than psychological wellbeing. No guidelines were found that specifically focused on the management of PND. Therefore PND was not formally assessed and detected within the clinics. In the absence of reliable scientific evidence and policy and guidelines, it is questionable whether the current approaches to promote and manage maternal mental health in Malaysia have a sense of cultural-based strategies. These significant gaps indicate the need for empirical evidence that explicitly focussed on the experiences and perceptions of PND in Malaysia. 


\section{REFERENCES}

Abdollahi, F., Etemadinezhad, S. \& Lye, M. S. (2014). Postpartum Mental Health in Relation to Sociocultural Practices. Taiwanese Journal of Obstetrics \& Gynecology, 55(1), pp 76-80.

Akmal, S. (2015). Traditional Postnatal Care In Restoring Women's Physical And Mental Health. Health Technology Assessment Section (Mahtas) Medical Development Division Ministry of Health Malaysia.

Azidah, A.K., Shaiful, B.I., Rusli, N. \& Jamil, M.Y. (2006). Postnatal depression and socio-cultural practices among postnatal mothers in Kota Bahru, Kelantan, Malaysia. Medical Journal of Malaysia, 61(1), pp 76-83.

Baby Center (2014a). Confinement Practices: an Overview. Retrieved from: http://www.babycenter.com.my/a1 021145/confinementpractices-an-overview

Baby Center (2014b). Indian Confinement Practices. Retrieved from: http://www.babycenter.com.my/ a1042118/indian-confinement-practices

Baby Center (2015). Antenatal care at government clinics, Retrieved from: http://www.babycenter.com.my/a1 049800/antenatal-care-at-government-clinics

Buultjens, M. \& Liamputtong, P. (2007). When giving life starts to take the life out of you: women's experiences of depression after childbirth. Midwifery, 23(1), pp 77-91.

Chee, C.Y.I., Lee, D.T.S., Chong, Y.S., Tan, L.K., Ng, T.P. \& Fones, C.S.L. (2005). Confinement and other psychosocial factors in perinatal depression: A transcultural study in Singapore. Journal of Affective Disorders, 89(1-3), pp 157-166.

Chong, S.T., Mohamad, M.S. \& Er, A.C. (2013). The mental health development in Malaysia: History, current issue and future development. Asian Social Science, 9(6), pp 1-8.

Country Report: Malaysia (2006). $4^{\text {th }}$ ASEAN \& Japan High Level Officials Meeting on Caring Societies: Support to Vulnerable People in Welfare and Medical Services. Retrieved from: http://www.mhlw.go.jp/bunya/ kokusaigyomu/asean/asean/kokusai/siryou/dl/h18_malaysia1.pdf

Cox, J.L., Holden, J.M. \& Sagovsky, R. (1987). Detection of postnatal depression. Development of the 10-item Edinburgh Postnatal Depression Scale. The British Journal of Psychiatry: The Journal of Mental Science, 150, pp 782-786.

Crabtree, S.A. \& Chong, G. (2001). Standing at the crossroads: mental health in Malaysia since independence. In: Haque, A., ed. Mental Health Issues in Malaysia, Issues and Concerns. Kuala Lumpur: University of Malaya Press.

Department of Statistics Malaysia. (2010). Population distribution and basic demographic characteristic report: Malaysian Government. Retrieved from: https://www.statistics.gov.my/index.php?r=column/ctheme\&menu _id=10pheu43nwjwrwvszklwdzq4tlhuut09\&bul_id=mdmxdhzjwtk1sjfztznkrxyzcvzjdz09

Deva, M.P. (2006). Depressive illness--the need for a paradigm shift in its understanding and management. The MedicalJournal of Malaysia, 61(1), pp 4-6.

Edhborg, M., Nasreen, H.E. \& Kabir, Z.N. (2015). "I can't stop worrying about everything"-experiences of rural Bangladeshi women during the first postpartum months. International Journal of Qualitative Studies on Health and Well-being, pages 9.

Fisher, J.R.W., Morrow, M.M., Ngoc, N.T.N. \& Anh, L.T.H. (2004). Prevalence, nature, severity and correlates of postpartum depressive symptoms in Vietnam. BJOG: An International Journal of Obstetrics and Gynaecology, 111(12), pp 1353-1360. 
Gao, L., Chan, S.W., You, L. \& Li, X. (2010). Experiences of postpartum depression among first-time mothers in mainland China. Journal of Advanced Nursing, 66(2), pp 303-312.

Grace, J., Lee, K.K., Ballard, C. \& Herbert, M. (2001). The relationship between post-natal depression, somatization and behaviour in Malaysian women. Transcultural Psychiatry, 38(1), pp 27-34.

Ishak, M. (2009). Cultural and religious tolerance: the Malaysian experience. International Conference on Migration, Citizenship and Intercultural Relations. Deakin University, Australia.

Jaafar, S., Suhaili, M., Mohd Noh, K., Ehsan, F. \& Lee, F. (2007). Malaysia: Primary health care key to intersectoral action for health and equity. World Health Organization and Public Health Agency of Canada. Retrieved from: http://www.who.int/social_determinants/resources/isa_primary_care_mys.pdf

Jamaiyah, H. (2000). Community mental health in Malaysia: Marriage of psychiatry and public health. Jurnal Kesihatan Masyarakat, 6(S), pp 155-166.

Kadir, A.A., Nordin, R., Ismail, S.B., Yaacob, M.J. \& Mustapha, W.M.R.W. (2004). Validation of the Malay version of Edinburgh Postnatal Depression Scale for postnatal women in Kelantan, Malaysia. Asia Pacific Family Medicine, 3(1-2), pp 9-18.

Kaur, J. \& Singh, H. (2011). Maternal health in Malaysia: A review. Webmed Central Public Health. 2(12). Retrieved from: https://www.webmedcentral.com/article_view/2599

Khader, F.R. (2012). The Malaysian experience in developing national identity, multicultural tolerance and understanding through teaching curricula: Lessons learned and possible applications in the Jordanian context. International Journal of Humanities and Social Science, 2(1), pp 270-288.

Kroenke, K., Spitzer, R.L. \& Williams, J.B. (2001). The PHQ-9: validity of a brief depression severity measure. Journal of General Internal Medicine, 16(9), pp 606-613.

Malaysia Nursing Board (2015). Nursing Board Malaysia Code of Professional Conduct for Nurses. Retrieved from: http://nursing.moh.gov.my/about-us/midwives-board/

Manap, M.A. (1975). The role of midwives and traditional birth attendants in maternal and child health and family planning in Malaysia. ICM/USAID Project West Asia Working Party, 17-25 October. Kuala Lumpur, Malaysia.

Ministry of Health Malaysia (2007). Clinical Practice Guidelines. Management of Major Depressive Disorder. Putrajaya: Ministry of Health Malaysia. Retrieved from: http://www.moh.gov.my/attachments/3897.pdf

Ministry of Health Malaysia (2008). The Kuala Lumpur declaration on primary health care 2008. National Conference on Primary Health Care Kuala Lumpur, Malaysia, Kuala Lumpur, Malaysia.

Ministry of Health Malaysia (2011). Psychiatric and mental health services operational policy. Putrajaya: Ministry of Health Malaysia. Retrieved from: http://www.moh.gov.my/images/gallery/Polisi/psychiatry_ operational_policy.pdf

Ministry of Health Malaysia (2012). Health Facts 2012. Putrajaya: Ministry of Health Malaysia. Retrieved from: http://www.moh.gov.my/images/gallery/stats/heal_fact/health_fact_2012_page_by_page.pdf

Ministry of Health Malaysia (2013). Midwives Board Malaysia: The code of professional conduct and practice of a midwife. Retrieved from: http://www.moh.gov.my/index.php/pages/view/1202

Ministry of Health Malaysia (2014). Guideline on Malay postnatal care, Traditional Complementary Medicine in Malaysia. Putrajaya: Ministry of Health Malaysia. Retrieved from: http://tcm.moh.gov.my/v4/pdf/handbook.pdf

Mitchell, A.J., Yadegarfar, M., Gill, J. \& Stubbs, B. (2016). Case finding and screening clinical utility of the Patient 
Health Questionnaire (PHQ-9 and PHQ-2) for depression in primary care: a diagnostic meta-analysis of 40 studies. British Journal of Psychiatry Open, 2(2), pp 127-138.

Morrow, M., Smith, J.E., Lai, Y. \& Jaswal, S. (2008). Shifting landscapes: immigrant women and postpartum depression. Health Care for Women International, 29(6), pp 593-617.

Mukhtar, F. \& Oei, T.P.S. (2011). A review on assessment and treatment for depression in malaysia. Depression Research and Treatment, pages 8.

Mustapha, F.I., Omar, Z.A., Mihat, O., Noh, K.M., Hassan, N., Bakar, R.A., Manan, A.A., Ismail, F., Jabbar, N.A. \& Muhamad, Y. (2014). Addressing non-communicable diseases in Malaysia: an integrative process of systems and community. BMC Public Health, 14(2).

Ng, C.G. (2014). A review of depression research in Malaysia. The Medical Journal of Malaysia, 69, pp 42-45.

Oei, T.P. \& Mukhtar, F. (2008). Exploratory and confirmatory factor validation and psychometric properties of the Automatic Thoughts Questionnaire for Malays (ATQ-Malay) in Malaysia. Hong Kong Journal of Psychiatry, 18(3), pp 92-101.

Razali, S. \& Hasanah, C. (1999). Cost-effectiveness of cyclic antidepressants in a developing country. Australian and New Zealand Journal of Psychiatry, 33(2), pp 283-284.

Sherina, M.S., Arroll, B. \& Goodyear-Smith, F. (2012). Criterion validity of the PHQ-9 (Malay version) in a primary care clinic in Malaysia. The Medical Journal of Malaysia, 67(3), pp 309-315.

Spitzer, R.L., Kroenke, K., Williams, J.B. \& Patient Health Questionnaire Primary Care Study Group (1999). Validation and utility of a self-report version of PRIME-MD: the PHQ primary care study. Journal of the American Medical Association, 282(18), pp 1737-1744.

Stahl, S.M. (2000). Essential psychopharmacology of depression and bipolar disorder. Cambridge university press, United Kingdom.

Stern, G. \& Kruckman, L. (1983). Multi-disciplinary perspectives on post-partum depression: an anthropological critique. Social Science \& Medicine, 17(15), pp 1027-1041.

Wan Mohd Rushidi, W.M., Amir A. \& Mahmood Nazar, M. (2003). Revalidation of the Malay version of the Edinburgh Postnatal Depression Scale (EPDS) among Malay postpartum women attending the Bakar Bata Health Center in Alor Setar, Kedah, Northwest of Peninsular Malaysia. Malaysian Journal of Medical Sciences, 10(2), pp 71-75.

Wittkowski, A., Zumla, A., Glendenning, S. \& Fox, J.R.E. (2012). The experience of postnatal depression in South Asian mothers living in Great Britain: a qualitative study. Journal of Reproductive \& Infant Psychology, 30(5), pp 480-492.

Yusuff, A.S.M., Tang, L., Binns, C.W. \& Lee, A.H. (2015). Prevalence and risk factors for postnatal depression in Sabah, Malaysia: a cohort study. Women and Birth, 28(1), pp 25-29. 